\title{
VISCOUS MHD FLOW ABOUT A SPHERICAL MAGNETIC QUADRUPOLE*
}

\author{
By VIVIAN O'BRIEN (Applied Physics Laboratory, The Johns Hopkins University)
}

\begin{abstract}
The perturbation velocity field and the perturbation magnetic field due to the first-order interaction of the slow flow field of a conductive viscous fluid with an aligned quadrupole magnet have been calculated. The drag increase can be computed from the perturbation stream function alone with an economy of effort compared to previous calculations for magnetized bodies.

Introduction. A steady axisymmetric magnetohydrodynamic (MHD) field allows the solenoidal velocity field of an incompressible fluid to be expressed in terms of a Stokes stream function $\psi$ and the corresponding solenoidal magnetic field in terms of a corresponding magnetic flux function $\Psi$ [1]. The complete set of normalized MHD equations reduces to two coupled non-linear equations:

$$
\begin{aligned}
& \mathscr{D}^{2} \psi=\operatorname{Re}\left\{\frac{-1}{r^{2} \sin ^{2} \theta} \frac{\partial(\psi, D \psi)}{\partial(r, \theta)}+\frac{2 D \psi}{r^{2} \sin ^{2} \theta}\left(\cos \theta \frac{\partial \psi}{\partial r}-\frac{\sin \theta}{r} \frac{\partial \psi}{\partial \theta}\right)\right\} \\
& +\frac{M^{2}}{R_{m}} \sin \theta\left\{\frac{\partial \Psi}{\partial r} \frac{\partial}{\partial \theta}-\frac{\partial \Psi}{\partial \theta} \frac{\partial}{\partial r}\right\} \frac{D \Psi}{r^{2} \sin ^{2} \theta}, \\
& D \Psi=\frac{R_{m}}{r^{2} \sin \theta} \frac{\partial(\psi, \Psi)}{\partial(r, \theta)},
\end{aligned}
$$

where $D=\partial^{2} / \partial r^{2}+r^{-2} \sin \theta(\partial / \partial \theta)\left[(\sin \theta)^{-1} \partial / \partial \theta\right]$ is the Stokesian operator, $\operatorname{Re}=U \alpha / \nu$ the Reynolds number, $M=\left(\sigma \alpha^{2} B^{2} / \rho \nu\right)^{1 / 2}$ the Hartmann number and $R_{m}=\mu \sigma \alpha U$ the Magnetic Reynolds number. $U$ is the steady uniform velocity at infinity, $\alpha$ the sphere radius, $\nu$ the kinematic viscosity, $\rho$ the density, $\sigma$ the electrical conductivity, $\mu$ the magnetic permeability of the fluid, $B$ the magnetic field intensity at the axis of the sphere, and $(r, \theta, \varphi)$ the spherical polar coordinate system. Contours of the $\psi$ field are streamlines and contours of the $\Psi$ field are magnetic lines of force. The relations of $\psi$ and $\Psi$ to the velocity and magnetic vector fields (definitions) are given by the component parts

$$
\begin{array}{lll}
u_{r}=\frac{1}{r^{2}} \frac{\partial \psi}{\partial(\cos \theta)}, & u_{\theta}=\frac{1}{r \sin \theta} \frac{\partial \psi}{\partial r}, & \left(u_{\varphi}=0\right), \\
h_{r}=\frac{1}{r^{2}} \frac{\partial \Psi}{\partial(\cos \theta)}, & h_{\theta}=\frac{1}{r \sin \theta} \frac{\partial \Psi}{\partial r}, & \left(h_{\varphi}=0\right) .
\end{array}
$$

The induced volume current field, $J i_{\varphi}^{*}$, is given by

$$
J=\frac{1}{r \sin \theta} D \Psi .
$$

Magnetic quadrupole solution. Assume small perturbations from the viscous velocity

*Received September 28, 1964; revised manuscript received December 22, 1964. This work supported by the Bureau of Naval Weapons, Department of the Navy, under Contract NOw 62-0604-C. 
field $\psi_{0}$ of a sphere in uniform flow given in [2] and small deviations from the magnetostatic field are given by the quadrupole flux function

$$
\Psi_{0}=r^{-2} C_{3}^{-1 / 2}(\cos \theta),
$$

where $C_{n}^{\gamma}(z)$ is a Gegenbauer polynomial of order $n$ and index $\gamma$ [1]. The field equations (1), can be linearized to two separate equations for the perturbation velocity field $\psi_{1}$ of $O\left(M^{2}\right)$ and the perturbation magnetic field $\Psi_{1}$ of $O\left(R_{m}\right)$. Satisfying the appropriate boundary conditions for real materials (viscous fluid, finite conductivity and permeability) the first-order MHD field is given by

$$
\begin{gathered}
\psi_{1}=M^{2}\left\{\frac{1}{28} C_{2}^{-1 / 2}(\cos \theta)\left[\frac{1}{9} r^{-4}-\frac{1}{18} r^{-5}-\frac{1}{594} r^{-7}-\frac{31}{(9)(33)} r^{-1}+\frac{5}{99} r\right]\right. \\
+\frac{1}{36} C_{4}^{-1 / 2}(\cos \theta)\left[r^{-4}-\frac{1}{14} r^{-5}+\frac{5}{143} r^{-7}-\frac{2927}{(14)(143)} r^{-3}+\frac{998}{(14)(143)} r^{-1}\right] \\
\left.+C_{6}^{-1 / 2}(\cos \theta)\left[-\frac{11}{126} r^{-4}+\frac{7}{143} r^{-5} \ln r+\frac{r^{-7}}{(13)(126)}+\frac{2411 r^{-5}}{(252)(143)}+\frac{713 r^{-3}}{(252)(143)}\right]\right\}, \\
\Psi_{1}=R_{m}\left\{C_{2}^{-1 / 2}(\cos \theta)\left[-\frac{3}{40} r^{-2}+\frac{1}{60} r^{-4}+\frac{7}{120} r^{-1}\right]\right. \\
\left.+C_{4}^{-1 / 2}(\cos \theta)\left[-\frac{2}{5} r^{-1}+\frac{7}{10} r^{-2}+\frac{1}{40} r^{-4}-\frac{13}{40} r^{-3}\right]\right\} .
\end{gathered}
$$

It is assumed that the magnetic permeability of the permanently magnetized body is effectively infinite, otherwise there would also be a disturbance magnetic field inside the sphere.

As in the non-magnetic velocity field calculations of [2], this "Stokes" solution $\psi_{1}$ overestimates the vorticity far ahead of the body through neglect of the inertial term in the governing equation. To complete the picture the "Stokes" solution should be matched to the appropriate "Oseen" solution in the manner of [2]. The matching is uniquely determined by the limiting form of $\psi_{1}$ as $r \rightarrow \infty$ but is not necessary here.

Drag. Usually the drag increase due to the Joulean dissipation of the induced currents flowing around the axis is computed by integrating the electromagnetic stresses over the unit sphere [3]. This electromagnetic drag increase is somewhat offset by a decrease in viscous dissipation from the non-magnetic case. The viscous drag change can be evaluated by integrating the viscous stresses over the sphere surface, but this first requires integration of the momentum equation to get the perturbation pressure field. However, the tractions of the MHD field can be evaluated on a very large control sphere after the manner of Goldstein [4]. By arguments similar to those advanced by Payne and Pell [5] in computing Stokes drag by a limit process, the total MHD drag can be computed from:

$$
\operatorname{Drag}_{M H D}=\lim _{r \rightarrow \infty} \frac{(8 \pi \rho \alpha \nu U) r \psi_{1}}{r^{2} \sin ^{2} \theta}=6 \pi \rho \alpha \nu U \frac{5 M^{2}}{4158} .
$$

Note this is the total MHD drag, not just the viscous contribution; it includes the effect of the electromagnetic stresses.

Conclusion. Similar calculations of $\psi_{1}$ and $\Psi_{1}$ for magnetic unipoles and magnetic dipoles (including matching to the "Oseen" solutions) have been carried out [6]. The 
drag results corroborate those published previously, $[3,7,8]$. Table I shows how rapidly the MHD drag effect falls off with increasing order of magnetic pole. Analysis for the higher order multipoles could be carried out in the same fashion, but, since the drag must be rather small, the computations have not been carried out.

TABLE I. MHD Drag of Spherical Magnetic Multipoles in Slow Uniform Flow.

\begin{tabular}{llccc}
\hline \hline Magnetic Pole & $\begin{array}{c}\text { Magnetostatic } \\
\text { Flux Function }\end{array}$ & $\frac{\text { Drag MHD }}{6 \pi \rho \alpha \nu U}=\begin{array}{c}\text { Electro } \\
\text { magnetic } \\
\text { Portion }\end{array}$ & $\begin{array}{c}\text { Viscous } \\
\text { Portion }\end{array}$ \\
\hline Unipole & $\Psi_{0}=C_{1}^{-1 / 2}(\cos \theta)$ & $\frac{37}{210} M^{2}$ & $\frac{1}{4} M^{2}$ & $-\frac{31}{420} M^{2}$ \\
Dipole & $\Psi_{0}=r^{-1} C_{2}^{-1 / 2}(\cos \theta)$ & $\frac{1}{150} M^{2}$ & $\frac{1}{60} M^{2}$ & $-\frac{1}{100} M^{2}$ \\
Quadrupole & $\Psi_{0}=r^{-2} C_{3}^{-1 / 2}(\cos \theta)$ & $\frac{5}{4158} M^{2}$ & $\frac{1}{252} M^{2}$ & $-\frac{163}{(63)(660)} M^{2}$ \\
Higher Multipole & $\Psi_{0}=r^{-n+1} C_{n}^{-1 / 2}(\cos \theta)$ & $\begin{array}{c}\sim 10^{-n} M^{2} \\
(\text { estimated })\end{array}$ & \\
\hline
\end{tabular}

\section{REFERENCES}

1. V. O'Brien, Axisymmetric magnetic fields and related problems, J. Franklin Inst. 275, 24-35 (1963)

2. I. Proudman and J. R. A. Pearson, Expansions at small Reynolds number for the flow past a sphere and a circular cylinder, J. Fluid Mech. 2, 237-262 (1957)

3. N. Riley, A magnetohydrodynamic Stokes flow, Proc. Royal Soc. A260, 79-90 (1961)

4. S. Goldstein, The forces on a solid body moving through viscous fluid, Proc. Royal Soc. A123, 216-225 (1929)

5. L. E. Payne and W. H. Pell, The Stokes flow problem for a class of axially symmetric bodies, J. Fluid Mech. 7, 529-549 (1960)

6. V. O'Brien, The first-order MHD flow about a magnetized sphere, APL/JHU Report CM-1011 (1962) and Slow viscous MHD flows about magnetized spherical bodies, APL/JHU Report CM-1047 (1964), (unpublished)

7. J. R. Barthel and P. S. Lykoudis, The slow motion of a magnetized sphere in a conducting medium, J. Fluid Mech. 8, 307-314 (1960)

8. K. Tamada, On the motion of a body carrying a magnetic field through a viscous conducting fluid, Tenth Internat. Congr. Appl. Mech. Stresa, 1960 